\title{
Multiple Mobile Robots Map Building Based on DSmT
}

\author{
Peng Li, Xinhan Huang, and Min Wang, Xiangjin Zeng \\ Intelligent Control and Robotics Laboratory, \\ Department of Control Science and Engineering, \\ Huazhong University of Science and Technology, Wuhan 430074, China \\ Email: lipeng_hubei@126.com
}

\begin{abstract}
In this paper, a new multi-robot system framework is introduced to solve the problem of multi-robot map building. Compared with the classical architectures introduced in the literature, this kind of framework enhances overall system performance, especially in reducing calculations and communications, improving real-time performance. Meanwhile a general basic belief assignment function (gbbaf) is constructed to deal with the uncertain and imprecise information obtained by sonar sensors with the application of new information fusion method DSmT (Dezert and Smarandache Theory) in the system and consideration of characteristics of sonar sensors. At last, Pioneer II mobile robots are used to carry out experiments of map building for both single-robot system and multi-robot system. The comparison of created ichnography with the real map testified the validity of DSmT and efficiency of multi-robot system for fusing imprecise information and map building proposed by this research.
\end{abstract} building

Keywords-DSmT, information fusion, multi-robot, map

\section{INTRODUCTION}

Efficient exploration of an entirely unknown environment is a great difficulty multi-robot system. Robots obtain information of unknown environment through their sensors such as sonar, laser, infrared, visual, etc. And sonar sensor, with its low price, convenient applicability and high efficiency of data processing, is usually adopted as the main sensor of robot.

Compared to the single-robot system, multi-robot system obtains more information and is more flexible, robust, timesaving in map building autonomous.

However, there are few appropriate system frameworks for multi-robot system to work under an entirely unknown environment. Traditional architectures are not suitable for this task. Moreover, due to the influence of darkness, obstacle, smog or other uncertain incidents, some robots may be lost because they can not "see" others. Vision system introduced in [1] or other detection means [2] are not suitable for the complicated environment.

Information fusion technology can perfectly solve these problems: imprecise environment information obtained from the entirely unknown environment and inaccurate, uncertain and even highly contradicted data acquired by sonar sensor. At present, there are many good fusion methods, such as probability theory [3], fuzzy theory [4], DST [5]. But these methods cannot deal with highly conflicting information like sonar data.

To solve mentioned difficulties, a brand new multi-robots system framework is constructed with the advanced information fusion method DSmT [6 8]. This framework is fully applicable to the exploration of multi-robots and map building with the improvement of system function and lessen of mass communication and calculation. Moreover, even in complicated environment where robots can not see each other, this system is still functional. The new method of DSmT is proposed by Jean Dezert (French) and Florentin Smarandache (American) in 2003. It can not only fuses imprecise and uncertain information but also perfectly deals with highly conflicting information, which provides powerful theoretical and practical tool for environment exploration of multi-robots.

\section{MULTI-ROBOT SYSTEM ARCHITECTURE}

Multiple mobile robots system is based on MAS (MultiAgent System). A multi-agent system is loosely-coupled network of problem solvers (agent) that work together to solve a given problem that is beyond their individual capabilities [3]. Multi-agent system is particularly suitable for supporting collaborative works [9], where knowledge of, and communication between, members of working teams are essential. Normally there are three types of MAS architecture, centralized architecture, distributed architecture and hybrid architecture.

The task for multiple mobile robots system to explore an unknown environment has three features as follows:

1) There is a large number of data. We must obtain environment information as much as possible for the purpose of mapping accurately.

2) It is real-time communication and there is a large amount of communications. Robots run in unknown environment, so we must monitor them to deal with possible sudden incidents. Thus, robots need to transport huge number of environment information to monitoring center timely.

3) There is a large amount of calculation when building map. Monitoring center must control robots in time, so it must fuse all information that obtained by all robots and build environment map in a very short time. Fusing process will cost a lot of time in a complex environment. 
4) In order to solve the three problems above, the architecture of multiple mobile robots system must enhance overall system performance, moreover it must be a real-time system, which has less calculation and compute efficiently. The traditional three architectures are not entirely suitable for these characters. So we introduce a new type of architecture named centralized-monitoring architecture. This architecture is an improvement of centralized architecture. The centralizedmonitoring architecture is shown as Fig. 1.

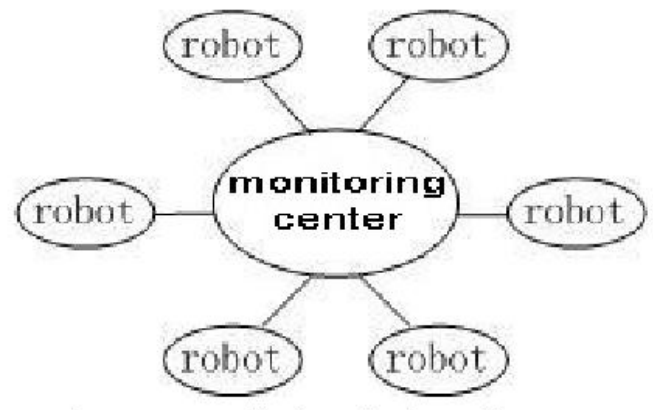

Figure 1. Centralized-monitoring architecture

This kind of architecture has the advantage of three traditional architectures. It is a simple real-time system. Each robot sends its environment data to monitoring center. After fusing these data, monitoring center builds a real-time global map and then controls all robots according to this map. In this kind of architecture, the system only needs to store a small amount of data, and monitoring center only needs to compute and fuse the data once in a communication cycle.

\section{COMMUNICATION ARCHITECTURE}

The communication architecture for multiple mobile robots system to explore unknown environment is shown as Fig. 2.

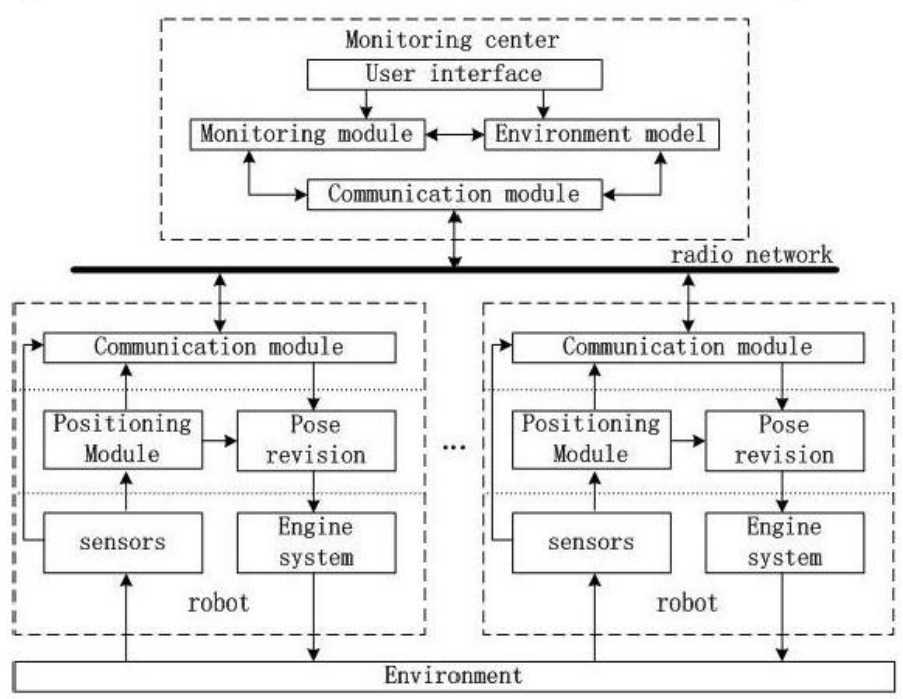

Figure 2. Multi-robot communication architecture

In this system, each robot obtains environment data by itself and transports the data to monitoring center through radio network. Monitoring center fuses these data, constructs the environment model and then sends commands to each robot.
Each mobile robot consists of three hardware modules (communication network system, sensor system and engine system) and three software modules (communication module, localization module and motion control module).

When the system works, firstly the monitoring center performs the connection to the robot. After the connection is established, the robot runs the processing cycle which is shown as follows:

1) Monitoring center sends command packets to robot through the connection and then robot carries out specified actions, such as avoiding obstacles, going to a point.

2) Every $100 \mathrm{~ms}$, localization module obtainsrobot's position and pose, sensor system collects data from the sensors.

3) Robot sends Server Information Packets (SIPs) to monitoring center through communication module. The standard SIP contains the robot's current position and estimates, current translational and rotational speeds, sonar reading updates, battery voltage, analog and digital I/O states, and more.

4) Once receives the data that sent from the robot, monitoring center creates the global map of environment and analyses robot's pose. According to the result of analysis, monitoring center sends adjustment command to robot.

5) Robot carries out the command of monitoring center and adjusts its pose. In the end, robot enters next cycle.

Multithreading synchronization technology is used to make the system work more efficiently. There are two threads, one is communication thread, and the other one is the computation thread.

\section{IMFORMATION FUSION OF COMMUNICATION-BASED MULTI-ROBOT SYSTEM}

\section{A. Fusion Structure}

The distributed fusion structure [10] of multiple mobile robot system is depicted in Fig. 3. To reduce the pressure on calculation of fusion center, all data obtained by robots must be reprocessed before fusing.

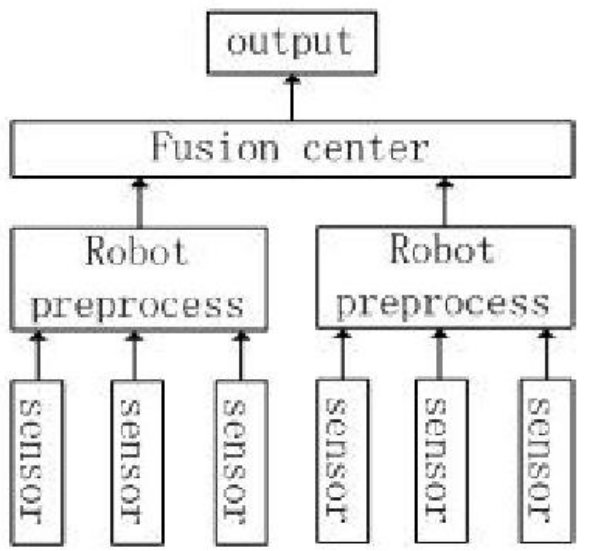

Figure 3. Distributed fusion structure of multi-robot system

\section{B. Fusion Algorithm}

The Dezert-Smarandache Theory (DSmT) of plausible and 
paradoxical reasoning proposed by the authors in recent years allows to formally combine any types of independent sources of information represented in term of belief functions. And it is mainly focused on the fusion of uncertain, highly conflicting and imprecise sources of evidence [11]. DSmT is able to solve complex static or dynamic fusion problems, specially when conflicts between sources become large and when the refinement of the frame of the problem under consideration, denoted $\Theta$, becomes inaccessible because of the vague, relative and imprecise nature of elements of $\Theta$.

\section{Simple Review of DSmT}

\section{1) Notion of hyper-power set $D^{\Theta}$}

One of the cornerstones of the DSmT is the notion of hyper-power set. Let $\Theta=\left\{\theta_{1}, \ldots, \theta_{n}\right\}$ be a finite set (called frame) of $n$ elements. The hyper-power set $D^{\Theta}$ is defined as the set of all composite propositions built from elements of $\Theta$ with $\cup$ and $\cap$ operators such that:

a) $\phi, \theta_{1}, \ldots, \theta_{n} \in D^{\Theta}$.

b) If $A, B \in D^{\Theta}$, then $A \cap B \in D^{\Theta}$ and $A \cup B \in D^{\Theta}$.

c) No other elements belong to $D^{\Theta}$, except those obtained by using rules $a$ ) or $b$ ).

\section{2) Generalized belief functions}

We define a map from a general frame $\Theta m(\bullet): D^{\Theta} \rightarrow[0,1]$ associated to a given source, say $\mathrm{B}$, of evidence as

$$
m(\phi)=0 \quad \text { and } \quad \sum_{A \in D^{\oplus}} m(A)=1
$$

The quantity $m(A)$ is called the generalized basic belief assignment/mass (gbba) of $A$.

The generalized belief and plausibility functions are defined as

$$
\begin{aligned}
& \operatorname{Bel}(A)=\sum_{\substack{B \subseteq A \\
B \in D^{\oplus}}} m(B) \\
& \operatorname{Pl}(A)=\sum_{\substack{B \cap A \neq \phi \\
B \in D^{\oplus}}} m(B)
\end{aligned}
$$

3) The Classic DSm Rule for Free-DSm Model

For $k$ independent uncertain and paradoxical sources of information providing generalized basic belief assignment $m_{i}(\bullet)$ over $D^{\Theta}$, the classical DSm conjunctive rule of combination [9] $m_{M^{f}(\Theta)}(A)$ is given by

$$
\begin{aligned}
\forall A \neq \phi \in D^{\Theta}, & \\
m_{M^{f}(\Theta)}(A) & \triangleq\left[m_{1} \oplus \cdots m_{k}\right](A) \\
& =\sum_{\substack{X_{1}, \cdots, X_{k} \in D^{\oplus} \\
\left(X 1 \cap \cdots \cap X_{k}\right)=A}} \prod_{i=1}^{k} m_{i}\left(X_{i}\right)
\end{aligned}
$$

$m_{M^{f}(\Theta)}(A)=0$ by definition, unless otherwise specified in special cases when some source assigns a non-zero value to it.

The conflict among the sets $\mathrm{X}_{\mathrm{i}}$ can be directly generalized for $\mathrm{k} \geqslant 2$ sources as follows:

$$
k_{12 \ldots s}=\sum_{\substack{X_{1}, \ldots, X_{s} \in D^{\oplus} \\ X_{1} \cap \ldots \cap X_{s}=\phi}} \prod_{i=1}^{s} m_{i}\left(X_{i}\right)
$$

The conflict mass must be distributed. Here we use PCR2 (Proportional Conflict Redistribution rule 2) to distribute the mass. The PCR2 [12] formula for $\mathrm{k} \geqslant 2$ sources is:

$$
\begin{aligned}
& \forall(X \neq \phi) \in D^{\Theta}, m_{P C R 2}(X)= \\
& {\left[\sum_{\substack{X_{1}, X_{2}, \ldots, X_{X} \in D^{\Theta} \\
X_{1} \cap X_{2} \cap \ldots \cap X_{s}=X}} \prod_{i=1}^{s} m_{1}\left(X_{i}\right)\right]+C(X) \frac{c_{12 \ldots s}(X)}{e_{12 \ldots s}} \cdot k_{12 \ldots s}}
\end{aligned}
$$

Where

$$
C(X)= \begin{cases}1, & \text { if } X \text { involved in the conflict, } \\ 0, & \text { otherwise; }\end{cases}
$$

In this formula, $c_{12 \ldots s}(X)$ is the non-zero sum of the column of $X$ in the mass matrix, $k_{12 \ldots .}$ is the total conflicting mass, and $e_{12 \ldots s}$ is the sum of all non-zero column sums of all non-empty sets involved in the conflict.

\section{Model of Sonar Sensor Based on DSmT}

The simple principle of sonar sensor is: it generates sheaves of cone-shaped wave to detect the objects, the wave will be reflected as soon as it encounters an object.

Since the sound wave generated by sonar sensor spreads forwards in the form of loudspeaker, there exists a divergence angle. Any objects among the fan-shaped can reflect the wave, so we cannot clearly know the real position of the objects detected among the fan-shaped area. And there are many other environment influences, so the data obtained by sonar sensor is not accurate.

We construct a model of sonar sensor (shown in Fig. 4.) based on DSmT to solve the problem. Suppose there are two elements $\theta_{1}$ and $\theta_{2}$ in the frame of discernment $\Theta$.

$\theta_{1}$ means the point in map is empty, $\theta_{2}$ means occupied, the hyper-power set is $D^{\Theta}=\left\{\phi, \theta_{1} \cap \theta_{2}, \theta_{1}, \theta_{2}, \theta_{1} \cup \theta_{2}\right\}$. Then we define $m\left(\theta_{1}\right)$ as the general basic belief assignment functions (gbbaf) for the empty status, define $m\left(\theta_{2}\right)$ as the gbbaf for occupied status, $m\left(\theta_{1} \cap \theta_{2}\right)$ is defined as the gbbaf of conflict mass, and $m\left(\theta_{1} \cup \theta_{2}\right)$ is defined as the gbbaf of unknown status (it mainly refers to those areas that still not be scanned at present). 


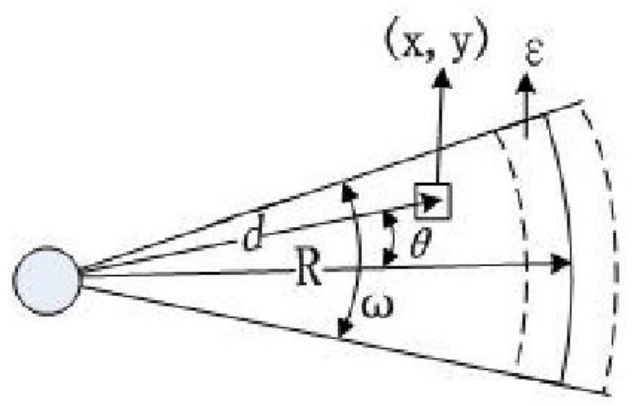

Figure 4. Sonar model based on DSmT

The belief assignments $m(\bullet): D^{\oplus} \rightarrow[0,1]$ are constructed by authors such as the formulas (7) (12) according to the sonar based on DSmT:

$m\left(\theta_{1}\right)=\left(1-\frac{\lambda}{2}\right) \cdot e^{\frac{-d^{2}}{2\left(R \rho_{E}\right)^{2}}}, \quad \mathrm{R}_{\min } \leq d \leq R+2 \varepsilon$

$m\left(\theta_{2}\right)=\lambda \cdot e^{\frac{-(d-R)^{2}}{2 R \rho_{O}^{2}}}, \quad \mathrm{R}_{\min } \leq d \leq R+2 \varepsilon$

$m\left(\theta_{1} \cap \theta_{2}\right)=e^{-\rho_{C}(\ln d-\beta)^{2}}, \quad \mathrm{R}_{\min } \leq d \leq R+2 \varepsilon$

$m\left(\theta_{1} \cup \theta_{2}\right)=$

$\begin{cases}(1-\lambda) \cdot \tanh \left(\frac{\rho_{I}(d-(R+\varepsilon))}{R}\right), \mathrm{R}+\varepsilon \leq d \leq R+2 \varepsilon \\ 0, \text { other }\end{cases}$

Where

$$
\begin{aligned}
& \beta=\ln \left(\frac{\rho_{E} \sqrt{R^{3}}}{\rho_{E} \sqrt{R}+\rho_{O}}\right) \\
& \lambda= \begin{cases}1-\left(\frac{2 \theta}{\omega}\right)^{2} & 0 \leq \theta \leq \frac{\omega}{2} \\
0 & \text { other }\end{cases}
\end{aligned}
$$

The equation (12) is given by [13]. Here $R_{\min }$ is the minimum sonar reading that sonar can obtain. $R$ is the sonar reading between sonar and object. $d$ is the distance from sonar to the point $(x, y)$ in the map. $\omega$ is the divergence angle, $\theta$ is the angle between $d$ and axis. $\varepsilon$ is the error of sonar reading. $\rho_{E}, \rho_{O}, \rho_{C}$ and $\rho_{I}$ are the environmental adjustment coefficients. Here we let $\rho_{E}=0.35, \rho_{O}=0.1$, $\rho_{C}=80$ and $\rho_{I}=10$. The analysis on the characteristics of gbbaf are shown as Fig. 5, when $\mathrm{R}=1.5$.

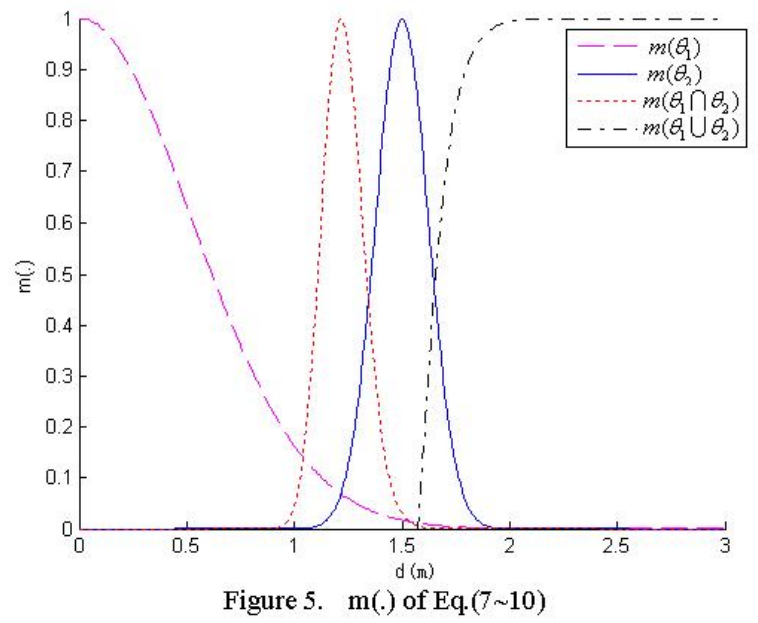

V. EXPERIMENTAL RESULTS AND ANALYSIS

We develop a user interface as a software platform for experiment by ourselves with Visual Studio 2005. The Pioneer II mobile robot that we use in experiments has 16 sonar sensors. Robot's structure is shown as Fig. 6 and the distribution of sonar sensors is shown as Fig. 7.

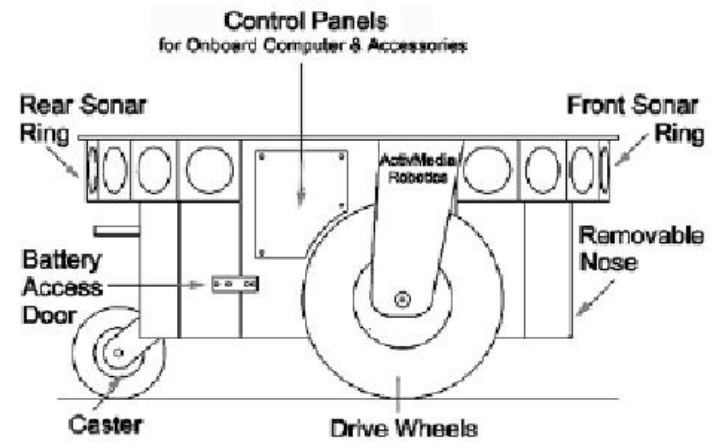

Figure 6. Robot structure

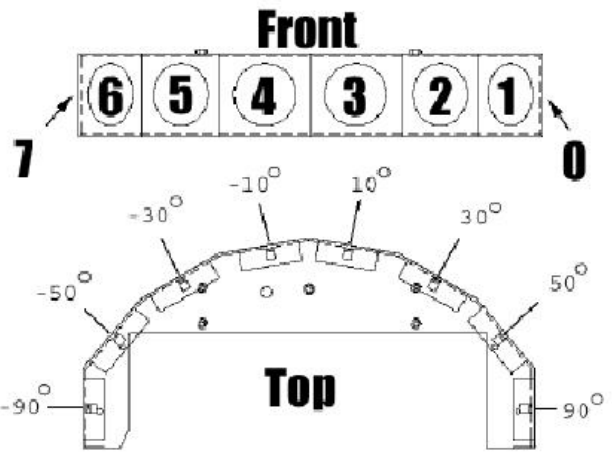

Figure 7. Distribution of front sonar sensors

First of all, the map of a room (size: $13 \times 8$ meters) is created. We carry out two experiments, one is for single robot in a runtime $t$, and the other is for multiple robots in the same runtime of $t$. The initial positions of multiple robots are shown as Fig. 8. The point of robot I is treated as the coordinate origin of the global map. So robot I is set to the pose of $\left(0,0,0^{\circ}\right)$, at 
the same time, robot II is set to the pose of $\left(6000,0,0^{\circ}\right)$. The third parameter is the deflection angle of robot.

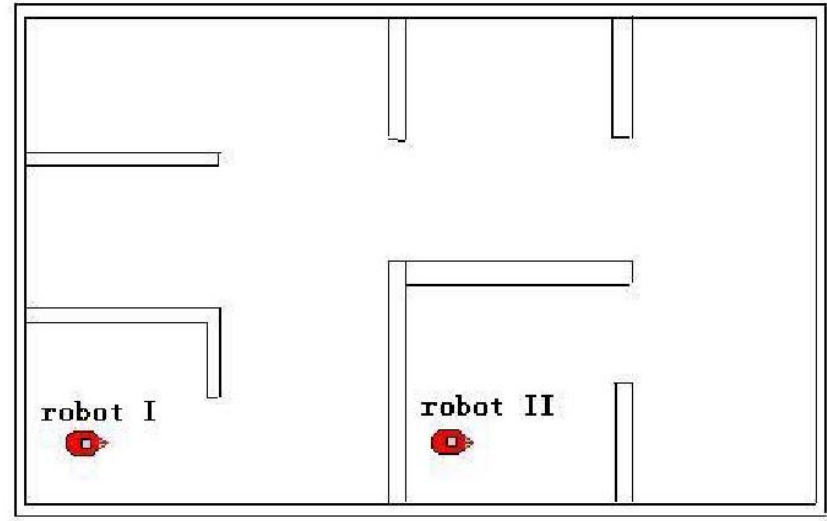

Figure 8. Initial positions of two robots

\section{A. Fusion Steps}

The data is fused in the monitoring center. The fusion steps are as follows:

1) Before exploring the environment, we propose the map is entirely unknown, that means $m\left(\theta_{1} \cup \theta_{2}\right)=1$;

2) Robots begin to explore the environment and send data to monitoring center. Monitoring center calculates the gbbaf $\left(m\left(\theta_{1}\right), m\left(\theta_{2}\right), m\left(\theta_{1} \cap \theta_{2}\right), m\left(\theta_{1} \cup \theta_{2}\right)\right)$ through(7) (12) and normalize them.

3) Apply the arithmetic of restrict spreading [14] to fuse the information in the fan-shaped area through (4) (5) and get new values of gbbaf.

4) Redistribute the conflict mass with PCR2 through (6).

5) Update values of gbbaf and go to step 2 .

6) Stop collecting data and exit at last.

\section{B. ExperimentalResults} 10.

The two experimental results are shown as Fig. 9 and Fig.

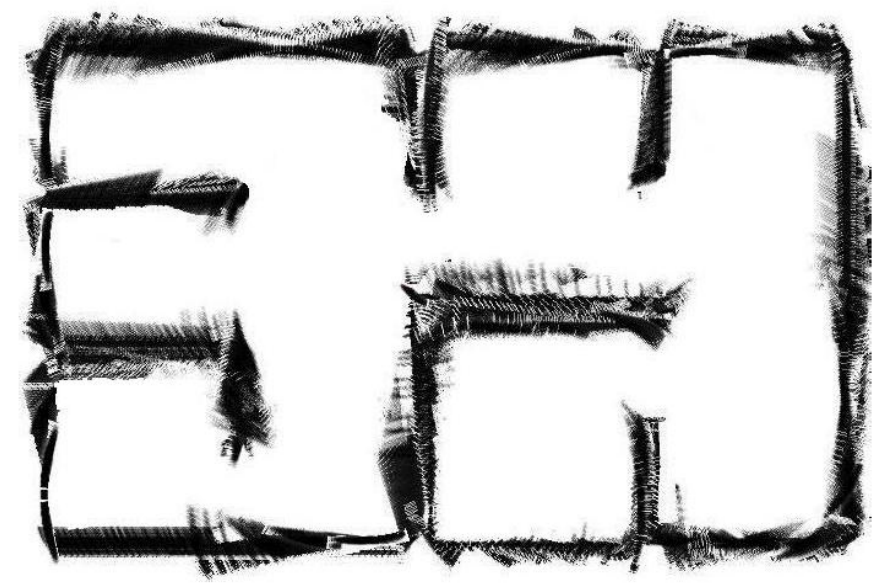

Figure 9 . The map built by a single robot

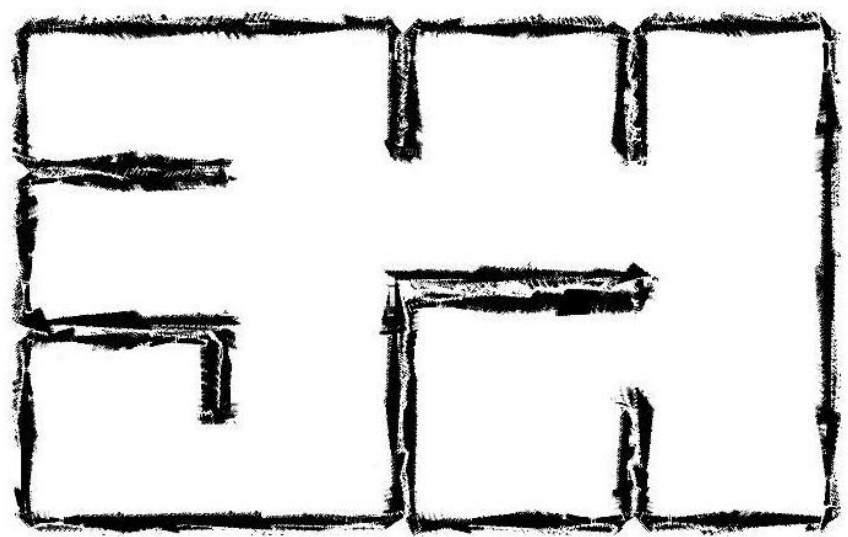

Figure 10. The map built by multiple robots

\section{Analysis}

1) We can apply DSmT to build accurate maps though the data obtained by sonar sensors is imprecise and uncertain. Outline of objects and location are expressed clearly.

2) As a result of Multithreading synchronization technology, the system works with out delay even breakdown.

3) Monitoring center can fuse information with high efficiency. Because of the application of DSmT, the fusion avoids the relatively complex division and only refers to simple addition and multiplication. And the arithmetic of restrict spreading makes us only need to update a small part of the map in one communication cycle.

4) Compared to single-robot system, multi-robot system not only can obtain more information, but also can fuse more information at the same time, so multi-robot system can describe the environment more accurately.

\section{CONCLUSION}

In this paper, we propose a framework for the development of multi-robot system. Compared with the classical architectures introduced in the literature, our multi-robot system architecture and communication architecture can make system work more efficiently and more stably. We also apply $\mathrm{DSmT}$ to mobile robot map building in a static virtual environment. Through the experiments, DSmT is proved to be a valid method, especially for multi-robot system to fuse imprecise information and build map accurately. To apply Hybrid DSmT [15] to multiple mobile robots system is our future work.

\section{ACKNOWLEDGMENT}

We are grateful to Dr. Jean Dezert from ONERA/DTIM, Chatillon, France for his guidance, help and technical supports.

This work was supported in part by the NSFC under grant No. 60675028 , this is greatly acknowledged.

\section{REFERENCES}

[1] A. K. Das et al., "A vision-based formation control framework," IEEE Transaction on Robotics and Automation, Vol. 18, No. 5, pp. 813-825, 2002. 
[2] S. Carpin, L. E. Parker, "Cooperative Leader Following in a Distributed Multi-Robot System," in Proceedings of IEEE Int. Conference Robotics and Automation, Washington, DC, pp. 2994-3001, 2002.

[3] W. Burgard, D. Fox, D. Hennig, T.Schmidt. Estimating the absolute position of a mobile robot using position probability grids. in: Procceeding of The National Conference on Artificial Intelligence (AAAI-1996). Oregon. 1996. 896 901

[4] Zadeh, L. Fuzzy sets. Information and Control,1965, 8(3): 338 353

[5] G. Shafer. A mathematical theory of evidence. Princeton. N.J: Princeton University press. 1976

[6] DEZERT J. Foundations for a new theory of plausible and paradoxical reasoning[J]. Informa tion and Secur ity, 2002, 9: 13 57.

[7] DEZERT J, SMARANDACHE F. On the generation of hyper-powersets for the DSmT: FUSION 2003: proceedings of the 6th Int. Conf.

[8] DEZERT J, SMARANDACHE F. Partial ordering of hyper-powersets and matrix rep resentation of belief functionswithin DSmT: proceedings of the 6th Int. Conf. on Information Fusion, Cairns, Queensland, Australia, 2003[C]. [S.1.]: [s.n], 2003: 1230-2003.

[9] L. Foner, Yenta: A Multi-Agent, Referral-based, Matchmaking System. Proceeding of the First International,Conference on Autonomous Agent, 1997.
[10] Bedworth M, O'Brien J.The Omnibus model:a new model of data fusion[J]. IEEE Tran. on Aerospace and Electronic Systems Magazine, 2000,15(4):30 36 .

[11] F. Smarandache, J. Dezert (Editors)( 2004), Advances and Applications of DSmT for Information Fusion, American Research Press, Rehoboth, Available: http://www.gallup.unm.edu/ smarandache/DSmT- book1.pdf

[12] F. Smarandache, J. Dezert (Editors). Advances and Applications of DSmT for Information Fusion (Collected works). Vol.2, American Research Press, Rehoboth, 2006. Web available: http://www.gallup.unm.edu/ ${ }^{\sim}$ smarandache/DSmT-book2.pdf.

[13] J. Dezert, A. Tchamova, T. Semerdjiev, P. Konstantinova.Performance evaluation of fusion rules for multitarget tracking in clutter based on generalized data association.in:Procceeding of the 8th Int. Conf. on Information Fusion. USA: Philadelphia, July 2005.2: 1 8

[14] WANGW. Map building by mobile robots in unknown environment [D] Shanghai: Shanghai Jiaotong University, 2003: 5234.

[15] F. Smarandache, J. Dezert, Advances and applications of DSmT for information fusion, Rehoboth: American Research Press, 2004. 